\title{
Percepción de las madres acerca del contenido de la información del diagnóstico de Síndrome de Down
}

\author{
LUCÍA TORRES G. ${ }^{1}$, EULÁliA MAIA C. ${ }^{2}$
}

1. Especialista en Psicología de la Salud y Alumna de Maestría en Ciencias de la Salud UFRN (Universidad Federal Río Grande do Norte).

2. Prof. Dra. de Postgrado en Ciencias de la Salud (UFRN) y del Curso de Psicología (UFRN). Coordinadora del Curso de Especialización en Psicología de la Salud: Desarrollo y Hospitalización (UFRN). Coordinadora de la base de investigación "Grupo de Estudios: Psicología y Salud - UFRN (Universidad Federal Río Grande do Norte).

\begin{abstract}
Mothers perception about information content in Down syndrome diagnosis

Objective: To analyze mothers perception about Down Syndrome (DS) diagnosis process, checking the quality of the information. Methods: Descriptive investigation with quantitative and qualitative focus, performed in 20 mothers from northeast Brazil through a semi-structured questionnaire. The data was analyzed by softwares SPSS (Stathistical Package for the Social Sciences) and ALCESTE 4.5 (Analyse Lexicale par Contexte d'un Ensemble de Segments de Texte), for Information Analysis and Thematic Content Analysis. The information was also analyzed quantitatively from categories elaboration. Inside the quantitative focus, the sociodemographic characterization was observed through software SPSS, in order to analyze simple frequency and the software ALCESTE, for the analytic treatment of the interview. The third item of the questionnaire was analyzed only by content analysis, because the number of words from the mothers answers were not enough for treatment by ALCESTE. Results: $90 \%$ of mothers received Down Syndrome diagnosis after delivery. $75 \%$ of cases were reported by pediatricians and $15 \%$ by nurses. Mothers said that the diagnosis was performed late, with inadequate and insufficient information and the interviewed mothers felt the same feelings reported in literature: shock, negation, sadness and anger, adaptation and reorganization. Conclusions: DS diagnosis was performed lately in the mayority of cases, concordant to Brazilian reality, principally when it concerns less priviledge economical classes. Mothers point that this late diagnosis, unsuitable and insufficient in information, produces feelings that the literature quotes as common: shock, negation, sadness and anger, adaptation and reorganization. In this way, we observed that the news can be a factor that difficult or facilitate the establishment of mother-son link, compromising the resource search for child development.

(Key words: Down Syndrome, diagnosis, communication, affection).

Rev Chil Pediatr 2009; 80 (1): 39-47
\end{abstract}

Trabajo recibido el 11 de enero de 2008, devuelto para corregir el 11 de marzo de 2008, segunda versión el 05 de mayo de 2008, tercera versión el 21 de julio de 2008, cuarta versión el 18 de diciembre de 2008, quinta versión el 30 de diciembre de 2008, aceptado para publicación el 12 de enero de 2009.

Correspondencia a:

Lucía María Torres G.

E-mail: loti_su@hotmail.com 


\section{RESUMEN}

Objetivo: Analizar la percepción de las madres acerca del proceso de información del diagnóstico de Síndrome de Down, buscando verificar la calidad de la información. Método: Investigación descriptiva, con enfoque cuantitativo y cualitativo y muestra de veinte madres del nordeste brasileño. El método investigativo fue un cuestionario semi-estructurado. Los dados fueron analizados por medio de los softwares SPSS (Statistical Package for the Social Sciences) e ALCESTE 4.5 (Analyse Lexicale par Contexte d'un Ensemble de Segments de Texte), que son softwares de análisis de información, y Análisis de Contenido Temático, y también fueron analizados cualitativamente, a partir de la elaboración de categorías. Dentro del enfoque cuantitativo, para la caracterización sociodemográfica se empleó el software SPSS, para análisis de frecuencia simple, y el software ALCESTE, para tratamiento analítico de las entrevistas. El ítem 3 del cuestionario fue analizado mediante análisis de contenido exclusivamente, ya que el número de palabras de las respuestas de las madres fue insuficiente para el tratamiento mediante ALCESTE. Resultados: 90\% de las madres recibieron el diagnóstico de Síndrome de Down después del parto. 75\% de los diagnósticos fueron comunicados por el médico pediatra y $15 \%$ por las enfermeras. Las madres refirieron que el diagnóstico fue tardío, inadecuado e insuficiente en lo informativo. Se observó que las entrevistadas vivieron los mismos sentimientos observados en la literatura como: shock, negación, tristeza e ira, adaptación y reorganización. Conclusión: Las madres perciben el diagnóstico como tardío, inadecuado e insuficiente en lo informativo, y genera sentimientos de negación, tristeza, ira, adaptación y reorganización. La comunicación del diagnóstico resulta un factor clave en la percepción de la enfermedad por las madres de pacientes con síndrome de Down.

(Palabras clave: Síndrome de Down; Comunicación, diagnóstico; Vínculo).

Rev Chil Pediatr 2009; 80 (1): 39-47

\section{Introducción}

El nacimiento de un niño acarrea alteraciones de la dinámica familiar ${ }^{1,2}$. El período posparto, normalmente, se describe como un momento de felicidad, no obstante, no es raro (entre 10 a $15 \%$ anualmente) que las madres entren en depresión ${ }^{1}$. Durante la gestación, la madre va preparándose para iniciar una nueva relación, que a veces puede conllevar gran discrepancia entre las expectativas imaginadas acerca del bebé y la realidad ${ }^{3}$.

Determinadas situaciones pueden comprometer seriamente el vínculo entre madre e hijo, como por ejemplo el nacimiento de un bebé con deficiencia, como Síndrome de Down (SD), 10 cual generalmente desencadena reacciones, sentimientos y comportamientos que pueden afectar profundamente la función materna. ${ }^{4} \mathrm{El}$ SD provoca un atraso en el desarrollo y generalmente se ve acompañado por algunos problemas de salud como cardiopatía congénita y obesidad $^{5}$. El SD representa aproximadamente $18 \%$ del total de deficientes mentales en instituciones especializadas ${ }^{6}$. Su incidencia en naci- dos vivos es de 1 en 600/800 nacimientos, con un promedio de 8000 nuevos casos por año en Brasil $^{7,8}$.

En ese contexto, los estudios que traten del impacto del diagnóstico se tornan importantes. Son diversos los estudios que analizan el impacto del diagnóstico ${ }^{9-15}$, sin embargo, persiste el uso de conceptos e informaciones inadecuadas, que producen una perspectiva negativa y comprometen la toma de decisiones futuras en relación a la vida de los hijos. ${ }^{15}$ Teniendo en vista las cuestiones precitadas, el objetivo de esta investigación fue analizar la percepción de las madres acerca del proceso de información del diagnóstico de SD, para verificar si las mismas consideran las informaciones recibidas suficientes o no. Por otra parte, se evaluó en qué momento, comúnmente, este diagnóstico es transmitido (antes o después del parto).

\section{Pacientes y Método}

Se trata de una investigación con enfoque cuantitativo y cualitativo, realizada en el municipio de Natal, en el Estado de Río Grande do 
Norte, Brasil, con una muestra de 20 madres cuyos hijos presentaron Síndrome de Down (SD), dentro de un rango etario de cero a tres años de edad, que recibieron atención en el Hospital de Pediatría (HOSPED) de la Universidad Federal de Río Grande do Norte (UFRN), en el período de febrero de 2005 a agosto de 2005. El criterio de inclusión fue: madres con hijos con diagnóstico de SD atendidas en el HOSPED que consintieron en participar en la investigación, mediante firma de un Consentimiento Informado y Libre (TCLE). La investigación fue aprobada por el Comité de Ética en Investigaciones de la Universidad Federal de Río Grande do Norte (protocolo de estudio número 194/96).

Para la colecta de datos, se utilizó un cuestionario semi-estructurado, aplicado individualmente, validado mediante estudio piloto, que fue realizado en madres con hijos con Síndrome de Down, de un centro de referencia de atención a niños con SD, en Río Grande do Norte. La validación fue efectuada a través de la Base de Investigación de Estudios de Psicología vinculada a la UFRN. Cabe destacar que el estudio piloto fue necesario para que el investigador verificase si los instrumentos de investigación o la metodología eran adecuados para el estudio $^{16}$.

La primera parte del cuestionario apuntó a caracterizar a las madres, incluyendo informaciones sociodemográficas como edad, ingresos, profesión y escolaridad de la pareja. La segunda parte cuestionó individualmente acerca de: 1) Cómo se transmitió la información: a) ¿A quién se transmitió la información del diagnóstico? b) ¿Quién transmitió esa información? c) ¿Cuándo se recibió la noticia? d) ¿Cómo fueron comunicadas tales informaciones al transmitirse el diagnóstico? e) ¿Esas informaciones fueron suficientes? Justifique; 2) Los sentimientos evidenciados al transmitirse la noticia: a) ¿Cuál fue el principal sentimiento evidenciado al comunicarse el diagnóstico?; y 3 ) Las expectativas (de las madres) en relación al futuro de sus hijos: a) ¿Qué expectativa tiene en relación a su hijo? Tales preguntas fueron empleadas para identificar la percepción de las madres frente al diagnóstico de Síndrome de Down de sus hijos.
Se realizó el análisis del material mediante un enfoque cuantitativo y cualitativo. Dentro del enfoque cuantitativo, para la caracterización sociodemográficas se empleó el software SPSS 13.0 (Statistical Pacckage for the Social Sciences), para análisis de frecuencia simple, y el software ALCESTE V 4.5 (Analyse Lexicale par Contexte d'un Ensemble de Segments de Texte), para tratamiento analítico de las preguntas del cuestionario ${ }^{17}$. Ese software permite el análisis léxico de un conjunto de datos textuales con el objetivo de distinguir clases de palabras en representación de las diferentes formas de discurso sobre el tema de interés. Combina las raíces semánticas definiéndolas por clases, teniendo en cuenta la función de la palabra dentro de un contexto determinado. Cada clase está compuesta de varias unidades de Contexto Escolar (UCE). Es computarizada para cada clase una línea de palabras que son características de la misma en la cual la fuerza de asociación entre cada palabra y su clase se expresa por un valor de chi-cuadrado $\left(\chi^{2}\right)$. Cuanto mayor el valor del $\chi^{2}$, más importante es la palabra para la construcción estadísticas de la clase. La lista de palabras es la fuente básica para la interpretación de las clases. Y para la interpretación del vocabulario específico de la clase y su respectiva temática, son consideradas las palabras con frecuencia igual o mayor $(\geq)$ que 3 e $\chi^{2} \geq 3,84(\mathrm{gl}=1)$. Importante recordar, que el software Alceste trabaja con frecuencia simple, porcentaje e asociación chi-cuadrado $\left(\chi^{2}\right)$.

Básicamente, ese programa ejecuta el análisis en 4 pasos, siguiendo criterios propios: $1^{\circ}$ paso: Organiza el material-las UCI (constará de las respuestas del cuestionario) formando un corpus. Por medio de una Análisis jerárquico descendente (ADH) - fragmenta el corpus en segmentos de textos similares, llamados de UCEs (Unidades de Contexto Elemental), para después combinarlas en funciones dos radicales e da frecuencia das palabras. $2^{\circ}$ paso: Clasifica las UCEs, a partir de una prueba de asociación-Chi-cuadrado $\left(\chi^{2}\right) .3^{\circ}$ paso: Hace el perfil de las clases. En el nivel de análisis, esas clases se componen de varios segmentos de texto (o UCEs) con vocabulario similares. En el nivel interpretativo son consideradas como 
indicadores de diferentes nociones, informando la distribución del texto analizado. En el caso de este estudio, el corpus fue distribuido en 5 clases léxicas (o clases de segmentos de texto), cuantificando la identificación de la percepción de las madres frente al diagnóstico de Síndrome de Down de sus hijos. Finalmente, el $4^{\circ}$ paso: Describe las UCEs más características de cada clase, permitiendo que se tenga disponible el contexto de la ocurrencia del vocabulario de las mismas, lo que permite diferentes interpretaciones en función de los objetivos de la investigación y las relaciones establecidas.

Frente a la complejidad del objeto de este estudio, se evidenció la necesidad de asociar técnicas complementarias y secuenciales a los datos cualitativos de la investigación. Se usó para ello una combinación cuantitativa y cualitativa de análisis: En primer lugar, efectuamos un análisis cuantitativo de los textos (análisis de palabras o respuestas, o análisis léxico) a partir del Alceste, y después, un análisis de contenido temático de Bardin (donde se identifican ideas según quien responde).

Puede decirse que el análisis léxico es el estudio científico del vocabulario, con aplicación de métodos estadísticos para la descripción del vocabulario permite identificar con mayor detalle las citas de los participantes, utilizando indicadores que relacionan aspectos a las citas y palabras ${ }^{18}$. Con el enfoque cualitativo, fundamentamos la integración de los descubrimientos, que trascienden lo aparente, dentro de un cuadro de referencia del todo social donde se dan los mensajes. Utilizamos el análisis temática de Bardin, donde: "El tema es la unidad significante que se libera naturalmente de un texto analizado, según criterios relativos a la teoría como guía de la literatura" 18 .

\section{Resultados}

Al momento de obtención de los datos (febrero de 2005 a agosto de 2005), eran 57 en total las madres con niños con SD atendidas en el hospital, de las cuales 20 aceptaron participar en la investigación.

Los sujetos entrevistados fueron madres de niños de 0 a 3 años de edad, con Síndrome de Down, con atención ambulatoria del Hospital Universitario. Tales madres se encontraban en un rango etario de 21 a 49 años, entre las cuales predominaron las mujeres de 37 a 44 años de edad (40\%). Las madres contaban con un nivel de escolaridad primario (40\%) y secundario (45\%). La principal ocupación de las entrevistadas consiste en servicios domésticos realizados en su propia casa (60\%), su mayoría viven con su pareja $(70 \%)$. Con respecto a los padres de los niños, $40 \%$ se encontraban dentro de un rango etario de 22 a 33 años (40\%), sin diferencias significativas entre las edades de los padres vs madres.

Se observa que existe una homogeneidad sociodemográfica de la populación estudiada, caracterizada por mujeres de clase media baja, con nivel de escolaridad primario a secundario.

Según sus compañeras, el nivel de escolaridad de los mismos es primario (45\%), variando bastante sus respectivas profesiones: el $35 \%$ de los padres son agricultores. La renta familiar de la muestra analizada se dividió en tres categorías, oscilando entre ingresos inferiores a un salario básico y seis salarios básicos $(70 \%)$.

En lo que se refiere a los niños, el $40 \%$ se encontraba en un rango etario de 1 a 9 meses de edad. Predominaron los niños de sexo masculino (60\%), siendo $40 \%$ de sexo femenino. El $60 \%$ de los niños tienen hermanos, y el 50\% del total constituyen los hijos menores de la familia. Todos los niños eran atendidos por la médica Pediatra del Hospital Universitario, y el 35\% sólo era atendido por tal profesional. También hubo gran incidencia de pacientes atendidos por dos $(25 \%)$ y tres especialistas médicos (20\%), si bien algunos niños eran atendidos por cuatro $(10 \%)$, cinco $(5 \%)$ e incluso nueve $(5 \%)$ especialistas de la salud.

En cuanto a quién transmitió la información del diagnóstico de Síndrome de Down, en el $75 \%$ de los casos fue comunicada por el médico pediatra y en un $15 \%$ fue transmitida por enfermeros o asistentes sociales. Tales profesionales no acompañaban a las pacientes anteriormente, ya que el Hospital de Pediatría (HOSPED) no ofrece atención prenatal. El HOSPED es una unidad adicional vinculada jerárquica y funcionalmente a la Rectoría de la 
Universidad Federal de Río Grande do Norte (UFRN), cuya misión es promover una formación universitaria con acciones asistenciales especializadas, preservando a los ciudadanos en el campo de la salud infantil. A nivel ambulatorio, el hospital ofrece diversos programas especializados, destacándose entre ellos el tratamiento de pacientes con SD (profesionales del programa: pediatra, fonoaudiólogo, fisioterapeuta, cardiólogo, nutricionista, neurólogo, psicólogo y asistente social, entre otros) y seguimiento ambulatorio a recién nacidos de riesgo. Por eso, las madres atendidas proceden de otros locales (hospitales, maternidades, dispensarios y unidades básicas, no sólo de Natal sino de todo el Estado), para atención especializada en pediatría.

Con respecto a lo que se considera aceptable para las madres con respecto al momento en que fue recibida la noticia del diagnóstico, en 1 caso se transmitió durante la gestación $(5 \%)$. En otro caso, el diagnóstico fue comunicado durante el parto $(5 \%)$, y en los demás casos ( $90 \%$ ) después, de los cuales el 20\% inmediatamente después del parto, $10 \%$ en las primeras 24 horas posteriores, $20 \%$ a los 3 días, $5 \%$ a los 18 días, $5 \%$ al mes y $5 \%$ a los 2 meses; el $35 \%$ restante no recuerda exactamente en qué momento recibieron la noticia después del parto. En cuanto a la calidad de la información, la mayoría de las madres $(65 \%)$ consideró que fue insuficiente, mientras que el 35\% restante consideró lo contrario. Por tratarse de un hospital de referencia pediátrica, la madre generalmente ya llega con el diagnóstico de su hijo, dado que esta unidad no dispone de ningún examen específico prenatal. Las madres son dirigidas al servicio del hospital para recibir atención especializada por un fonoaudiólogo, fisioterapeuta, cardiólogo, nutricionista, neurólogo, psicólogo o asistente social, entre otros.

Con respecto a los sentimientos experimentados por las madres al comunicarse el diagnóstico, 30\% manifestaron shock ante la noticia, $20 \%$ negación, $13 \%$ tristeza, $12 \%$ ira, 5\% adaptación, $4 \%$ reorganización y $16 \%$ no supieron responder.

En lo que se refiere a las expectativas de las madres en relación al futuro de sus hijos, los datos revelaron que $66 \%$ de las madres consi- deran que sus hijos dependerán efectivamente de ellas para sobrevivir, 19\% estiman que sus hijos conseguirán desarrollarse, pero precisarán de alguna ayuda de los padres o familiares, $10 \%$ creen que sus hijos podrán desarrollarse y vivir de una forma totalmente independiente de ellas o de la familia, y 5\% no supieron responder.

Según la prueba Chi-cuadrado $\left(\chi^{2}\right)$, hubo una asociación entre la forma en que la información fue dada en la transmisión del diagnóstico, con la expectativa que la madre asume en relación a su hijo. En un nivel de confianza del $99 \%$, el p-valor fue 0,00072 . Por lo tanto, se rechaza la posibilidad de que no hay asociación entre las dos variables.

En el tratamiento analítico de las respuestas abiertas del cuestionario, cuanto a la identificación de la percepción de las madres frente al diagnóstico de Síndrome de Down de sus hijos, el corpus analizado por el software ALCESTE $^{17}$ fue compuesto de 129 unidades de contexto inicial -UCIs- o respuestas del cuestionario (Etapa A1). La investigación del diccionario (el cálculo de las palabras por sus raíces o por sus matrices, Etapa A2) presentó 487 palabras o formas distintas o diferentes; 1469, UCEs Unidades de Contexto Elemental, u ocurrencias; frecuencia media de una palabra igual a 3. En la selección de las UCEs que sean consideradas (Etapa B1), se obtuvieran 46 palabras para análisis, 46 palabras instrumentales y 7 palabras con asterisco (variables). Las 46 palabras para análisis ocurren 340 veces. Em la definición o descripción de las clases escogidas (Etapa $\mathrm{C} 1$ ), se obtuvieron 5 clases o UCEs en la cual 72 UCEs fueran clasificadas de 132 seleccionadas, o sea, $54,55 \%$ del total.

Las clases serán descritas en su número de UCE, porcentaje dentro del total de las UCEs del corpus, la variable estadísticamente asociada y la temática inferida a partir del análisis del contenido, respectivamente. De esta forma, la clase 1 presentó 18 UCEs $(25 \%$, dificultades y sentimientos de las madres en el momento del diagnóstico; Inseguridad y preocupación en el cuidado), la clase 2 con 11 UCEs $(15,28 \%$; Sentimiento actual de las madres después del diagnóstico; Tristeza por lo que las personas irán pensar); la clase 3 con 10 UCEs $(13,89 \%$; 
Expectativas de las madres cuanto al futuro de los hijos; Esperanza); la clase 4 con 21 UCEs (29,17\%; Cualidad del diagnóstico transmitido; Información tardía y contenido insuficiente/inadecuado del diagnóstico) y la clase 5 con 12 UCEs $(16,67 \%$; Facilitadores de la relación madre-niño; Aceptación de la madre).

En cuanto a la cualidad del diagnóstico transmitido, el contenido de la clase 4 está destinado a verificar si las madres consideran las informaciones suficientes o no y es una de las clases más importantes en función del número total de UCEs (21 UCEs o $29,17 \%$ del total de las UCEs clasificadas). Recordemos que una clase está formada por un conjunto de UCEs y palabras (o radicales) más frecuentes (mayor valor de $\chi^{2}$ ) contenidos en tal clase, y optamos por utilizar las palabras más importantes. Cuanto mayor es el valor de chi-cuadrado $\left(\chi^{2}\right)$, más significativa resulta la palabra en la clase correspondiente. Así, el foco de la clase alude a la cualidad del diagnóstico transmitido, subdividido en dos categorías: categoría Informaciones tardías del diagnóstico y categoría Contenido insuficiente e inadecuado del diagnóstico, evidenciándose 6 elementos que contextualizan los discursos: Andar $\left(\chi^{2}=12,00\right)$, Dijo $\left(\chi^{2}=\right.$ 17,76), Hablar $\left(\chi^{2}=24,98\right)$, Médico $\left(\chi^{2}=20,53\right)$, Down $\left(\chi^{2}=13,05\right)$, Síndrome $\left(\chi^{2}=20,80\right)$.

En la categoría Informaciones tardías del diagnóstico, se presenta los siguientes relatos: "[...] a los tres días la médica dijo que él era especial [...] el pediatra dijo que sospechaba que tenía síndrome, entonces procuré saber [...] (ACM-madre entrevistada)". "[...] La médica no dijo nada [...]. La gente decía que era diferente. Me enteré concretamente cuando fui al pediatra, ella ya tenía 1 (un) año de edad. Ahí fue que comencé a ocuparme [...] (COL-madre entrevistada)". Analizando las respuestas acerca de cómo se transmitió el diagnóstico de Síndrome de Down, se observa que ante la falta de informaciones adecuadas hubo atraso en la busca de atención especializada para los niños, como se ve en los relatos maternos acima. Es interesante observar en ese contexto que para las madres el médico debería aparecer como profesional capacitado para ofrecer informaciones completas sobre el síndrome, pero no fue lo ocurrido.
En la categoría Contenido insuficiente e inadecuado del diagnóstico, se presenta los siguientes relatos: "[...] usted sabía que su hijo es enfermo? Tiene sindrome de Down [...] (POS-madre entrevistada)". "[...] El médico dijo: Su hijo es mogólico [...] (BPL-madre entrevistada)". "[...] Esa criatura no va a poder hablar, no va a caminar [...] me desesperé... no sabía qué hacer... no conocía a nadie asi [...] (UAT-madre entrevistada)". La insuficiencia de informaciones o frustración con respecto al proceso vinculado al diagnóstico también se describe en la literatura ${ }^{10-11}$.

\section{Discusión}

Las investigaciones acerca de la comunicación del diagnóstico de SD van ganando espacio en la literatura nacional e internacional ${ }^{2,9-13}$. La forma en que se comunica el diagnóstico puede contribuir o no para que el vínculo madre-bebé sea favorable. Los resultados del presente estudio fueron semejantes a los de otros que tratan la cuestión del diagnóstico de SD. El rango etario de las madres con hijos con diagnóstico de SD en el presente estudio fue similar a los de la literatura ${ }^{19}$. Los estudios revelan que si bien normalmente cabe al médico comunicar a los padres el diagnóstico ${ }^{2}$, como ocurrió en la presente investigación, los profesionales de enfermería desempeñan un papel importante, ya que son ellos los que tienen mayor contacto con la madre y el bebé .

En el presente estudio, la mayoría de las madres comunicaron que recibieron el diagnóstico de SD después del parto $(90 \%)$. Generalmente, el diagnóstico posparto desencadena tensión, angustia y estrés, junto con decepción, shock, ira ${ }^{2}$, entre otros, y miedo ${ }^{10}$, que suelen aparecer frente a la situación. En nuestro estudio, los sentimientos frente al diagnóstico de SD fueron semejantes a los destacados en la literatura, dado que los más notables fueron shock, negación, tristeza e ira, adaptación y reorganización. Sin embargo, estudios realizados muestran que cuando el diagnóstico se realiza después del parto, el procedimiento más adecuado es dar la noticia acerca de la deficiencia dentro de las primeras 24 a 48 horas posteriores al nacimiento. Ese plazo fue establecido por los 
mismos padres al evaluar retrospectivamente tal período, pues consideran que la madre ya está restableciéndose del parto, ya vio al bebé y comenzó a amamantar ${ }^{20}$. Los investigadores alegan que al crearse ya un vínculo afectivo a partir de esas acciones, existe mayor probabilidad de comprensión y aceptación del diagnóstico. En este estudio, sólo $10 \%$ de las madres recibió el diagnóstico dentro de las 24 horas posteriores al nacimiento y $35 \%$ lo recibió al cabo de 48 horas.

En lo que se refiere a la noticia del diagnóstico, la literatura evidencia que después del parto la misma desencadena tensión ${ }^{2-10}$, angustia y estrés, junto con decepción, shock, ira, principalmente cuando no existe una preparación adecuada de las madres ${ }^{2}$. En la presente investigación, la mayoría de las madres $(20 \%)$ recibieron el diagnóstico de SD de sus hijos tras el parto. Skotko ${ }^{10}$, describió un resultado semejante, tras realizar una investigación en los Estados Unidos en individuos de 5 organizaciones de padres con hijos con síndrome de Down. El cuestionario de esta investigación fue enviado a 2945 personas, de las cuales $1250(42,04 \%)$ lo respondieron. Entre ellas, 985 eran madres que recibieron el diagnóstico después del parto. El autor también indica que la mayoría de esas madres informaron que al recibir el diagnóstico sintieron "miedo" o ansiedad, y una minoría transmitió que la experiencia fue positiva en su totalidad. En ese sentido, la importancia del diagnóstico de SD en la fase prenatal es de suma importancia, ya que en esa fase los padres pueden ser preparados para la llegada del hijo con SD, existiendo la posibilidad además, de ser dirigidos a grupos de apoyo, que se describen como benéficos para el bienestar de la familia ${ }^{9,11}$. Debe destacarse que la llegada de un nuevo miembro en la familia normalmente 'desorganiza' la estructura familiar, que a partir de ese momento tendrá que adaptarse a la llegada de ese nuevo miembro. Cuando se trata de la llegada de un hijo con diagnóstico de SD, tal realidad se torna aún más compleja, ya que el mismo requerirá cuidados especiales.

Acerca de la cualidad del diagnóstico (insuficiente e inadecuado) un estudio realizado en madres con hijos con diagnóstico de SD procuró investigar de qué forma y en qué el proceso de diagnóstico prenatal podría ser mejorado. Se enviaron 2945 cuestionarios a personas de cinco organizaciones de padres con hijos con SD, de los cuales 1126 cuestionarios fueron respondidos. Entre ellos, 141 (12\%) correspondieron a madres que recibieron el diagnóstico durante la fase prenatal. Si bien se mostraron satisfechas con la atención que recibieron, la mayoría indicó que sintió frustración durante el proceso ${ }^{12}$. Esta frustración con respecto al proceso de diagnóstico de SD también fue descrita por Skotko ${ }^{10}$ en otra investigación que involucró 5 organizaciones de padres con hijos con SD, donde en la mayoría de los casos las madres recibieron el diagnóstico posparto. Esas madres relatan que sintieron que los médicos hablaron poco de los aspectos positivos del SD y raramente disponibilizaron materiales impresos sobre el asunto, o teléfonos de otros padres con hijos con SD, para poder intercambiar ideas.

Si bien los padres tienen derecho a saber lo que ocurre con sus hijos, algunos profesionales aún omiten informaciones adecuadas acerca del síndrome, observándose la ausencia de cualquier tipo de información comunicada a las madres en el momento del diagnóstico. Aunque no exista un modelo único que pueda utilizarse a la hora del diagnóstico, algunas actitudes son recomendables: transmitir el diagnóstico a los padres tan pronto como sea posible, observando sus condiciones emocionales para recibirlo; la comunicación debe ser realizada por una persona con conocimiento actualizado y que inspire confianza; además, el diagnóstico debe ser informado a los padres en lugar privado, discretamente, sin interrupciones y con tiempo suficiente para aclarar las dudas de los progenitores. El vocabulario utilizado debe ser simple y accesible $^{21}$. A partir del diagnóstico, por lo tanto, los padres enfrentan la diferencia entre el hijo deseado y el hijo real, siendo necesario elaborar el duelo del ideal perdido y encarar los sentimientos de frustración, rabia y decepción.

Evidenciamos que las madres del estudio refieren una insuficiencia en cuanto a las informaciones del diagnóstico de SD, no sólo por haber sido éste comunicado en la mayoría de los casos posparto, sino también por la presencia de falta de información y/o falta de conteni- 
do concreto y científico del síndrome, que podría ayudar a los padres a comprender mejor cómo lidiar con el problema de su progenie. Se infiere, a partir de ese momento, que la "información" puede ser interpretada como factor que dificulta y/o facilita en cuanto a la búsqueda de recursos para el niño.

En los relatos de algunas madres se observa el shock, tristeza, ira, entre otros al ver sus expectativas frustradas, frente a un hijo diferente, así como la desestabilización familiar consiguiente ocurrida en algunos casos. Tales sentimientos también son citados en la literatura como comunes en madres que se deparan con un diagnóstico de $\mathrm{SD}^{10,11}$. Todo eso resulta agravado por las informaciones erradas, inadecuadas, que a veces reciben. Es importante que las madres vuelvan a sus hogares con conocimiento del diagnóstico, pese a que muchas veces lo reciben de forma altamente destructiva ${ }^{8}$. Tal contenido insuficiente puede generar concepciones erróneas con respecto al síndrome, lo cual lleva frecuentemente a que las madres no confíen en el desarrollo de sus hijos. Esta afirmación se corrobora cuando observamos que el $66 \%$ de las madres creen que sus hijos dependerán efectivamente de ellas para sobrevivir. Y como sabemos, actualmente el progreso de los tratamientos permite cada vez más el desarrollo de los niños con SD, siendo cada vez mayor el número de personas con $\mathrm{SD}$ que trabajan y consiguen mantenerse, dentro de sus limitaciones. Inclusive, hoy en día existen en Brasil políticas de incentivo a empresas para la contratación de personas con necesidades especiales, lo cual está permitiendo el ingreso de esas personas en el mercado de trabajo.

Aunque el shock sea inevitable ${ }^{2}$, actitudes como una orientación adecuada y constructiva sobre el síndrome y sus implicaciones, así como sobre las posibilidades existentes y los caminos a recorrer, hacen que la inseguridad se reduzca y que esas madres pasen a buscar alternativas de atención compatibles ${ }^{15}$, favoreciendo de esa forma el desarrollo normal de sus hijos. La primera reacción puede ser de shock $^{2,15}$, porque es difícil comprender lo ocurrido, ya que las primeras imágenes que los padres forman del niño se fundan en los significados atribuidos anteriormente a la deficiencia, lo cual dificultará sobremanera la calidad de los cuidados a seguir a partir de ese primer momento.

Pese a la variación entre una familia y otra, cuando la asistencia es satisfactoria, gradualmente comienzan a establecerse vínculos afectivos más profundos con el niño, principalmente entre madre y bebé, así como la búsqueda de recursos en la comunidad para asistirlo, factores esenciales para el desarrollo del bebé. Incluso porque, pese a que el shock ante el diagnóstico de SD sea inevitable, especialmente para la madre, normalmente la mayoría de las familias supera la crisis y alcanza un equilibrio. ${ }^{2}$ Así, el diagnóstico de SD puede tornar-se más positivo ${ }^{13}$, dependiendo de la forma en que el diagnóstico es transmitido a la familia y de cómo se maneja la atención médica ofrecida al niño, ya que la colaboración de los profesionales puede minimizar el impacto, mostrando posibilidades positivas y no sólo aspectos negati$\operatorname{vos}^{2,12-13}$. En un estudio realizado en padres con hijos con diagnóstico de SD, las principales sugerencias de tales padres para mejorar el diagnóstico se refirieron al hecho de que el diagnóstico sea comunicado personalmente, que se disponga de materiales impresos para los padres con informaciones sobre SD, y que las madres sean dirigidas hacia grupos de apoyo a padres con SD. El estudio concluye que el hecho de recibir el diagnóstico de SD en fase prenatal no debe ser una experiencia negativa. A través de la implementación de las sugerencias propuestas, la atención puede tornarse más positiva $^{11}$.

Vislumbramos la necesidad de invertir en la capacitación de los profesionales que tratan de forma más directa con el diagnóstico de SD, para que el mismo sea dado abordando no sólo los aspectos negativos, sino también los positivos. También vislumbramos la necesidad de que el diagnóstico sea dado ya en la fase prenatal, que las madres puedan ser acompañadas por un equipo especializado en la fase posparto, y que la atención se prolongue hasta el momento en que se sientan fortalecidas y capacitadas para acompañar el desarrollo de sus hijos. 


\section{Referencias}

1.- Tammentie T, Paavilainen E, Astedt-Kurki P, Tarkka $M T$ : Family dynamics of postnatally depressed mothers - discrepancy between expectations and reality. J Clin Nurs 2004; 13: 65-74.

2.- Sunelaitis RC, Arruda DC, Marcom SS: The impact of a Down Syndrome diagnosis on the family dynamic: mother's perspective. Acta Paul. Enferm. 2007 [cited 2007 Nov 11]; 20(3): 264-271. Disponível em: http://www.scielo.br/scielo.

3.- Braselton TB: O desenvolvimento do apego: uma família em formação. Porto Alegre: Artes Médicas; 1988.

4.- Klaus M, Klaus PO: Surpreendente recém-nascido. Porto Alegre: Artes Médicas; 1989.

5.- Opitz JM, Gilbert-Barness EF: Reflections on the pathogenesis of Down syndrome. Am J Med Genet 1990; 7 (Suppl): 38-51.

6.- Moreira LMA, El-Hani CN, Gusmão FAF: A síndrome de Down e sua patogênese: considerações sobre o determinismo genético. Rev Bras Psiquiatr [periódico na Internet]. 2000 Jun [citado 2007 Dez 07]; 22 (2): 96-99. Disponível em: http://www.scielo.br/scielo.

7.- Moeller I: Diferentes e Especiais. Rev Viver Mente e Cérebro 2006; 156: 26-31.

8.- Schwartzman JS: Síndrome de Down. São Paulo: Mackenzie, 1999.

9.- Lenhard W, Breitenbach E, Ebert H, SchindelhauerDeutscher HJ, Zang KD: Henn Attitudes of mothers towards their child with Down syndrome before and after the introduction of prenatal diagnosis. WIntellect Dev Disabil 2007; 45: 98-102.

10.- Skotko BG: Mothers of Children With Down Syndrome Reflect on Their Postnatal Support. Pediatricts 2005; 115; 64-77.
11.- Skotko BG: Prenatally diagnosed Down syndrome: mothers who continued their pregnancies evaluate their health care providers.Am J Obstet Gynecol 2005; 192: 670-7.

12.- Van Riper M: Maternal perceptions of family-provider relationships and well-being in families of children with Down syndrome. Res Nurs Health 1999; 22: 357-68.

13.- Hall S, Bobrow M, Marteau TM: Psychological consequences for parents of false negative results on prenatal screening for Down's syndrome: retrospective interview study. BMJ 2000; 320: 407-12.

14.- Martins LAR: A inclusão escolar do portador de síndrome de Down: o que pensam os educadores? Natal: EDUFEN/UFRN; 2002.

15.- Macedo $B C$ : Um olhar das mães sobre os filhos com Síndrome de Down e seu processo evolutivo. (Dissertação). Natal (RN): Universidade Federal do Rio Grande do Norte; 2000.

16.- Köche JC: Fundamentos de metodologia científica: teoria da ciência e prática da pesquisa. Ed. Porto Alegre: Vozes, 2001, p. 23-39.

17.- Reinert M: Une méthode d'analyse des données textuelles et une application: Aurelia de G. De Nerval. Bulletin de Méthodogie Sociologique 1990; 26: 24-54.

18.- Bardin L: Análise de Conteúdo. Tradução de Luiz Antero Reto e Augusto Pinheiro. São Paulo: Martins Fontes; 1977.

19.- Cunningham C: El síndrome de Down: una introducion para padres. Barcelona: Paidós/ Fundació Catalana Síndrome de Down; 1990.

20.- Castilla EE, Lopes-Camelo JS, Paz JE: Atlas de las Malformaciones Congénitas en Sudamérica. Rio de Janeiro: Editora Fiocruz; 1995.

21.- Casarin S: Aspectos psicológicos na síndrome de Down. In: Schwartzman, JS. Síndrome de Down. São Paulo: Mackenzie 1999; 263-85. 